\title{
Analytic discrete cosine harmonic wavelet transform based OFDM system
}

\author{
M N SUMA $^{1, *}$, S V NARASIMHAN ${ }^{2}$ and B KANMANI ${ }^{1}$ \\ ${ }^{1}$ BMS College of Engineering, Bull Temple Road, Bangalore 19, India \\ ${ }^{2}$ CSIR-National Aerospace Lab, PB 1779, Old Airport Road, Kodihalli, \\ Bangalore - 560 017, India \\ e-mail: Suma.bms@gmail.com; narsim@nal.res.in
}

MS received 20 October 2013; revised 29 August 2014; accepted 21 October 2014

\begin{abstract}
An OFDM based on Analytic Discrete Cosine Harmonic Wavelet Transform (ADCHWT_OFDM) has been proposed in this paper. Analytic DCHWT has been realized by applying DCHWT to the original signal and to its Hilbert transform. ADCHWT has been found to be computationally efficient and very effective in improving Bit Error Rate (BER) and Peak to Average Power Ratio (PAPR) performance. Improvement compared to that of Haar-WT OFDM and DFT OFDM is achieved without employing Cyclic Prefix BER is 0.002 for ADCHWT OFDM compared to Haar WT, DFT OFDM which have BER of 0.06 and 0.4, respectively, at $15 \mathrm{~dB}$ SNR. PAPR is also reduced by $3 \mathrm{~dB}$ compared to DFT OFDM and $0.3 \mathrm{~dB}$ reduction compared to Haar WT OFDM.
\end{abstract}

Keywords. Analytic discrete cosine harmonic wavelet transform (ADCHWT); OFDM; dual tree complex wavelet transform; DCT; shift invariant wavelet transform; PAPR.

\section{Introduction}

Orthogonal Frequency Division Multiplexing is an efficient method of transmission with high bandwidth efficiency which is achieved by employing orthogonal and overlapped subcarriers (Prasad 2004). In practise, OFDM is digitally implemented by employing Discrete Fourier Transforms (DFT) as basis. DFT being a complex orthogonal transform employs FFT algorithm which is computationally efficient. Drawback of this implementation is rectangular windowing, which creates high side lobes resulting in leakage effect. Application of wavelets in signal processing as an alternative to Fourier basis has been suggested for multicarrier transmission (Lakshmanan \& Nikookar 2006; Thomas Weickert et al 2009; Narasimhan et al 2012). Wavelet filters have sharp stop-band attenuation and hence there are no side lobes. Wavelet Transforms

${ }^{*}$ For correspondence 
(WT) can be realised in time domain employing perfect reconstruction dyadic filter banks or by employing harmonic wavelet transform in frequency domain by (Narasimhan et al 2012). Wavelet OFDM does not require any cyclic prefix (CP) and hence it has bandwidth efficientlty (Lakshmanan \& Nikookar 2006). Absence of CP improves WT-OFDM bandwidth efficiency by about $20 \%$ as compared to that of DFT-OFDM (Lakshmanan \& Nikookar 2006; Mirghani \& Ghavami 2008).

WT implemented by a perfect reconstruction filter bank (PRFB) involves analysis filter bank, decimation, interpolation and synthesis filterbank. Decimation of the subband using Discrete Wavelet transform (DWT) suffers from shift-variance and oscillating coefficients. Shiftinvariance is achieved by either undecimated WT or Dual tree complex WT (Michael Bauer et al 2009). Dual-tree complex wavelet transform (DTCWT), a shift-invariant transform is proposed for image processing (Selesnick et al 2005). Real and imaginary parts of the coefficients are calculated using two independent filter bank trees, increasing computational cost by a factor of two, as compared to real wavelet transform. Fourier transform (FT) has a smooth magnitude response and provides shift invariance; however leakage effect persists. Realization of DTCWT employing Analytic Wavelet Transform (AWT) will provide advantages of both WT and FT (Selesnick et al 2005; Shivamurti \& Narasimhan 2010).

AWT is realized employing dual tree filter bank structure and Hilbert transform (HT). Analytical and complex-valued functions are employed, instead of real-valued wavelet basis functions to form the DTCWT. AWT has two parallel filter banks for analysis as well as for synthesis (Selesnick et al 2005; Michael Bauer et al 2009; Shivamurti \& Narasimhan 2010). One filter bank represents the real part of the transform while the other represents the imaginary part (HT of input). This reduces aliasing and accounts for the decimation by a factor of 2 at each DWT stage, leading to shift invariance. WT realized by a two channel PRFB in time domain has higher processing complexity due to explicit decimation, interpolation, associated filtering and delay compensation in reconstruction (Narasimhan et al 2012; Shivamurti \& Narasimhan 2010).

Discrete Cosine Harmonic wavelet transform (DCHWT) has been successfully employed for spectral estimation and compression (Narasimhan et al 2008). In order to realise the benefits of harmonic wavelet transform, DCT is employed in place of DFT. Employing DCT has lowered leakage effect while providing smooth transition from one signal period to the other, without discontinuity. A new DCHWT OFDM for BPSK and QPSK modulated signals have been discussed in our previous work (Suma et al 2012). The new system has an improved BER and PAPR performance, compared to DFT-OFDM. Its performance is on par with that of time domain Haar WT OFDM system. DTCWT OFDM proposed by Mohammed Nerma et al 2009 in, has given a $3 \mathrm{db}$ reduction in PAPR; however, all the said processing complexities remain with conventional wavelet transforms.

It would be interesting to explore HWT features to form ADCHWT OFDM that inherits all advantages of DCHWT and provides shift invariance for transmission in fading channels. This paper proposes the use of ADCHWT to overcome the problem of shift variance and can be employed as an alternate to undecimated WT for OFDM. This method has no leakage effect and requires no additional processing steps. It has shown improved performance compared with conventional OFDM for exponential channel model (Jeruchim et al 2000).

The Paper is organised in four sections. In section 2, ADCHWT and scale formation in DCHWT are discussed. In section 3, description of block model of the proposed ADCHWT OFDM is discussed. Section 4 deals with performance analysis indices. Section 5 deals with simulation results. Conclusions are provided in section 6. 


\section{Analytic discrete cosine harmonic wavelet transforms (ADCHWT)}

Entire bandwidth available in DCHWT OFDM is divided into orthogonal and overlapping subbands/scales. Data is assigned to wavelet scales having different time-frequency resolutions. ADCHWT realised by DTCWT will employ two trees; one for the real part and the other for imaginary part i.e., the Hilbert Transform of the input. Corresponding scales of the two trees are combined to achieve desired ADCHWT. Uniqueness of this transform lies in that it neither requires any complex filters nor suffers from any performance limitation. Dual tree approach of ADCHWT is illustrated for four scales in figure 1. DCHWT of four scales for the original signal are first obtained; HT is applied to input signal and again its DCHWT of four scales are obtained. Hilbert transformed scales are weighed by $j$ and are combined with their corresponding scales by summation to get the ADCHWT signal shown in figure 1 .

Subcarrier formation in DCHWT for DCT size of $\mathrm{N}=16$, with only one side of the symmetrical coefficient sequence i.e., (0-7) is shown in figure 2. Last half parts of the coefficients $\mathrm{x}$ (1) to $\mathrm{x}$ (7) correspond to scale-0, i.e., C4. Lower half of the coefficients $\mathrm{x}(0)$ to $\mathrm{x}(3)$ are split into two groups and the upper group $[\mathrm{x}(2), \mathrm{x}(3)]$ correspond to scale-1, C3. Lower group $[\mathrm{x}(0)$, $\mathrm{x}(1)]$ is further split into two groups $\mathrm{C} 2$ and $\mathrm{C} 1$ each having single coefficients $\mathrm{x}(1)$ and $\mathrm{x}(0)$, respectively.

For reconstruction, the DCTs of the subband signals $C 3, C 2, C 1, C 0$, are concatenated to get the DCT of the full-band signal (figure 2); its inverse DCT provides the reconstructed signal. At the transmitter end of OFDM, inverse transform is applied to subbands that are concatenated and transmitted. At the receiver, forward transform is applied to get back to DCT domain and subbands are decomposed as shown in figure 2.

\section{The proposed ADCHWT OFDM}

An OFDM based on Analytic Discrete Cosine Harmonic Wavelet Transform (ADCHWT_ OFDM) has been proposed in this paper. Block diagram of baseband transmitter and receiver of ADCHWT OFDM is shown in figure 3. To obtain scales for OFDM, dual tree complex wavelets

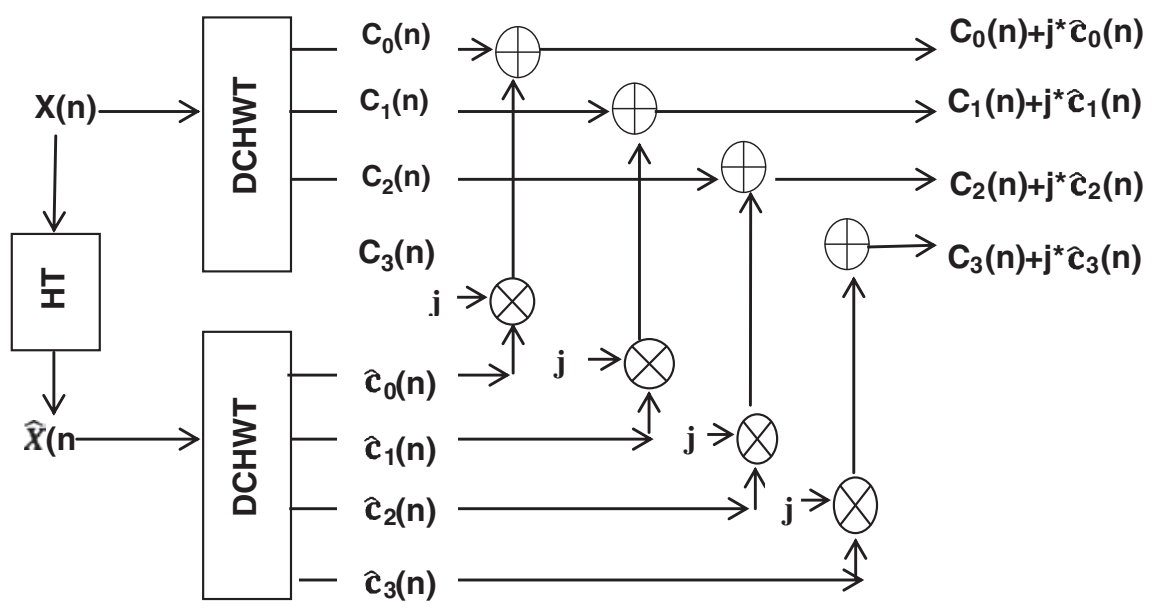

Figure 1. Schematic of ADCHWT formation. 

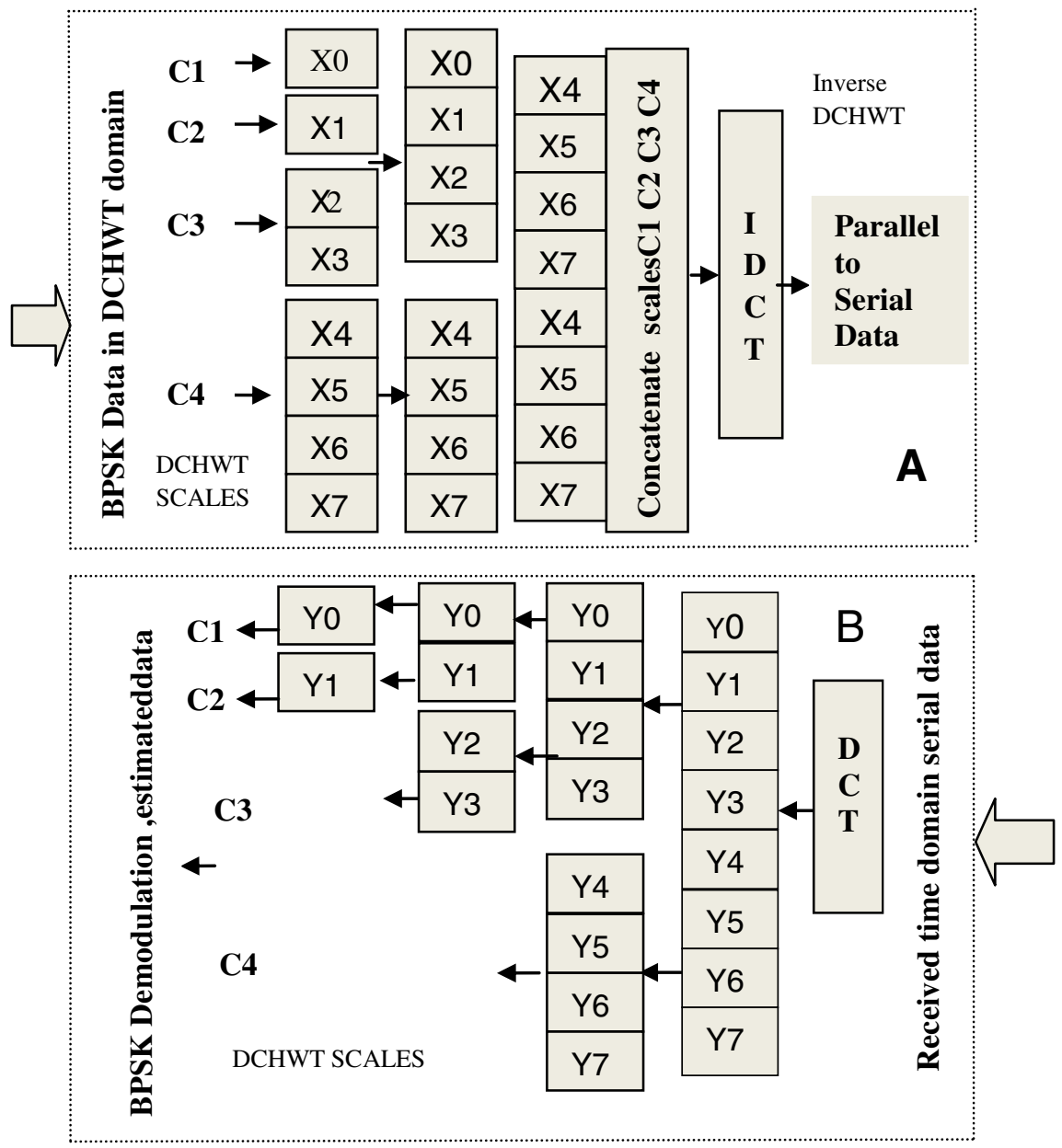

Figure 2. Schematic of DCHWT scale formation and reconstruction for OFDM.

transform employs two trees, as discussed in section 2. Corresponding scales of two trees are combined to achieve the desired ADCHWT OFDM subbands.

At the transmitter, incoming random bit stream is transformed to DCHWT domain by BPSK constellation mapping to form real part. HT is applied to the copy of the same bit steam and mapped again into BPSK constellation to form imaginary part. Real and imaginary parts are then applied to two separate DCHWT trees (figure 3). Real DCHWT scales formed are [C0 C1 C2 C3] and the imaginary scales are $\left[\hat{c}_{0}(n) \hat{c}_{1}(n) \hat{c}_{2}(n) \hat{c}_{3}(n)\right]$. IDCT is applied to these scales and added to form OFDM signal which is then transmitted in time domain. Employing wavelet domain eliminates the need for $\mathrm{CP}$, due to time overlap of symbols. An added advantage in harmonic wavelet domain is reduction of process complexities. Transmitted OFDM signal is given by

$$
x[n]=\sum_{k} \sum_{p=0}^{N-1} x_{k p} \psi_{p}(\mathrm{n}-k N),
$$




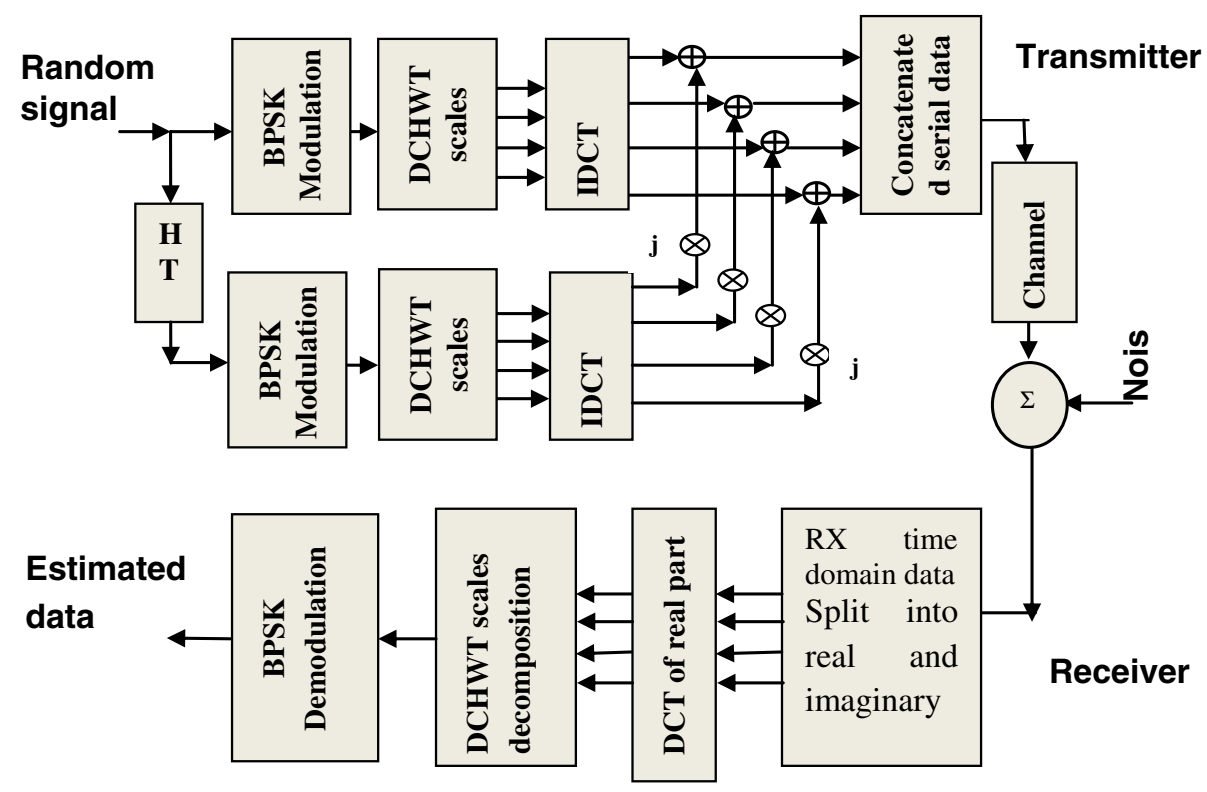

Figure 3. Block diagram of ADCHWT OFDM.

where $x_{k p}$ is a constellation of $\mathrm{k}^{\text {th }}$ data symbol modulating the $\psi_{p}(\mathrm{n})$ is the wavelet basis, which is the cosine modulated sinc function.

At the receiver end, the received time domain signal, corrupted due to channel is separated into real and imaginary parts. DCT is applied to the real part of time domain signal to form DCHWT scales [C0, C1, C2, C3] by subband decomposition. BPSK demodulation is then performed by Maximum Likelihood (ML) method to estimate bits transmitted.

\section{Performance indices for analysis}

Bit Error Rate (BER) and Peak to Average Power Ratio (PAPR) are the performance indices considered for comparison of the proposed ADCHWT OFDM system.

\subsection{Bit error rate}

Modulation schemes of the subcarriers are generally Quadrature Amplitude Modulation (QAM) or Phase Shift Keying (PSK). BER for the exponential indoor channel model with additive white Gaussian noise (AWGN) can be written in a general form as Q-function.

$$
\mathrm{BER}=\mathrm{Q}\left(\mathrm{g}\left(\frac{\sigma_{\mathrm{s}}^{2}}{\sigma_{n}^{2}}\right)\right),
$$

$\sigma_{\mathrm{s}}^{2}$ and $\sigma_{\mathrm{n}}^{2}$ are variance of signal and noise, $\mathrm{g}$ is constant, where $\mathrm{Q}(y)=\frac{1}{2 \pi} \int_{y}^{\infty} e^{-t^{2} / 2}$.

Difference in estimated and transmitted bits gives number of bit errors, which when divided 
by total bits sent, provides the bit error rate. Error probability curves are plotted for different SNR's from 0 to $30 \mathrm{~dB}$.

\subsection{Peak to average power ratio (PAPR)}

PAPR is one of the issues that need to be addressed in OFDM implementation. Complementary Cumulative Distribution Function (CCDF) of PAPR is a performance index which indicates the probability of statistically independent samples of OFDM that exceed the specified threshold value. PAPR values of wavelet OFDM system for $N$ sub-carriers depend on the nature of the wavelet basis used and the oversampling factor $L$. PAPR is given by

$$
\operatorname{PAPR}[x(n)]=10 \log _{10} \frac{N . \max _{0 \leq n \leq N L-1}\left\{\left|x_{s k}(n)\right|^{2}\right\}}{E\left\{\left|x_{s k}(n)\right|^{2}\right\}},
$$

where, $\mathrm{N}=$ number of subcarriers, $\mathrm{L}=$ Oversampling factor, $x_{s k}(n)$ is BPSK symbol.

PAPR reduction is achieved, for the proposed method without any additional compensation techniques.

\section{Simulation and discussion}

Parameters employed for simulation are listed in table 1. MATLAB is employed for simulation. Exponential channel is given by

$$
h(l)=e^{-\sigma^{2} *(l+1)} \quad 0 \leq l \leq M,
$$

Values of $\sigma=0.8$ and length $\mathrm{M}=5$ are employed for simulation.

There are many possible models for channels that can be selected for simulation depending on the applications. Exponential diffuse channel model is often used for comparative performance evaluations of different schemes in practise. Generally, for exponential models of indoor wireless simulation, channel profile length (M) of 5 is considered.

\subsection{BER Performance}

Performance of DCHWT-OFDM has been compared with those of Haar WT-OFDM and DFTOFDM for BPSK (figure 4). At $15 \mathrm{~dB}$, Eb/No BER achieved for ADCHWT OFDM is 0.002 which is a considerable improvement compared to Haar and DFT OFDM, which have BER of 0.06 and 0.4 , respectively. It is also seen that even at lower Eb/No, WT OFDMs has better performance than DFT OFDM.

Table 1. Simulation parameters.

\begin{tabular}{lc}
\hline Modulation type & BPSK \\
\hline Type of OFDM basis & DCHWT, Haar, DFT \\
Input stream & 4096 \\
Oversampling factor L & 4 \\
Tree decomposition depth for WT & 3 \\
Subcarrier size for DFT(N) & 512 \\
\hline
\end{tabular}




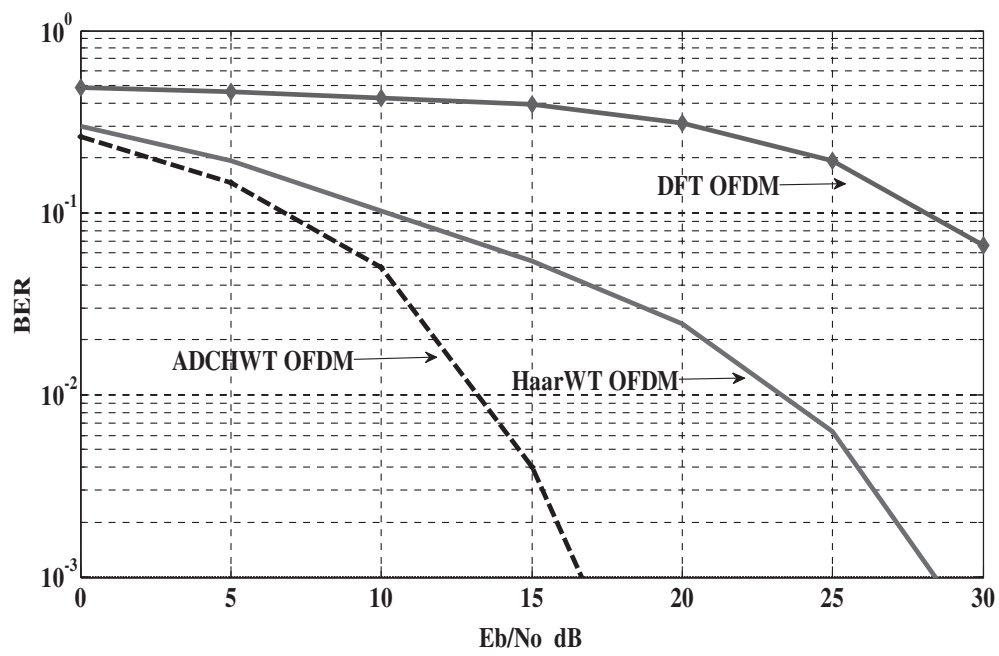

Figure 4. BER performance for ADCHWT, HAAR WT, DFT OFDM.

\subsection{PAPR Performance}

The proposed ADCHWT-OFDM provides PAPR reduction when compared to DFT-OFDM (figure 5). At CCDF of 0.001 , PAPR is reduced by $3 \mathrm{~dB}$ with the proposed method as compared to DFT OFDM. Compared to Haar WT OFDM, $0.3 \mathrm{db}$ reduction is achieved; this indicates PAPR distribution is in par with Haar WT OFDM. Time waveform (figure 6), indicates larger peaks for conventional OFDM than ADCHWT OFDM, which supports this improvement. It would be interesting to note, that the above performance improvement is achieved with processing simplicity.

5.2a Processing simplicity: Comparison of processing simplicity is shown in table 2. Processing in time domain wavelets include decimation, interpolation, filtering and delay compensation.

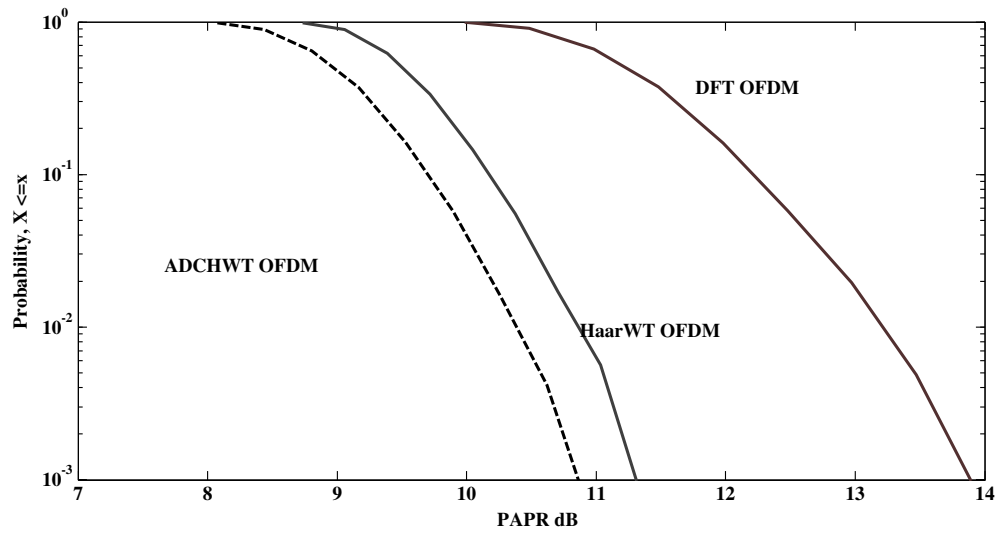

Figure 5. CCDF plots for ADCHWT, HAAR WT, DFT OFDM. 


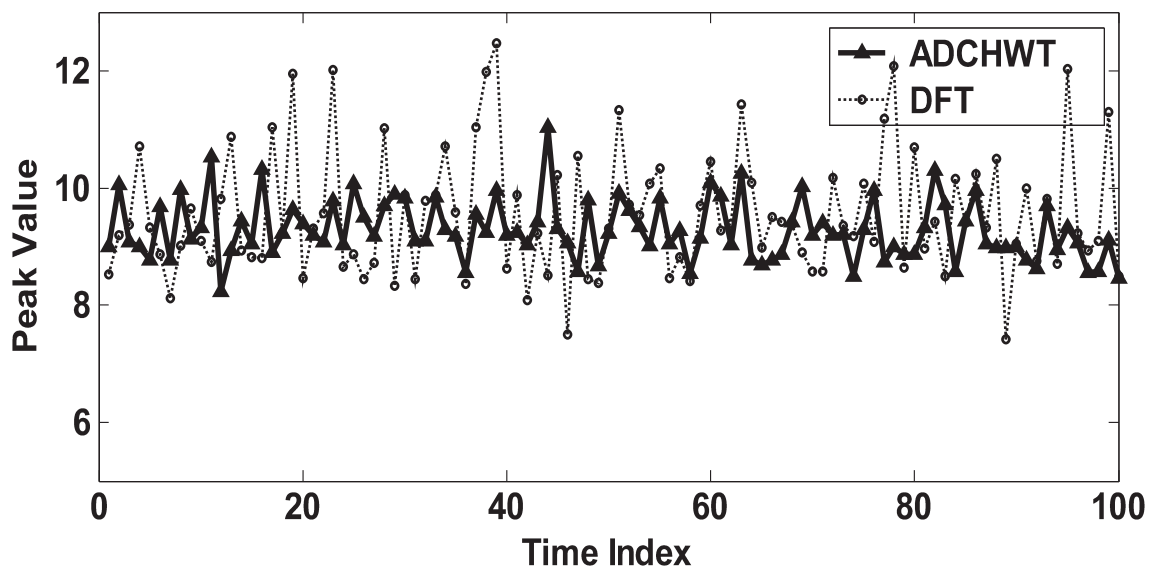

Figure 6. Time plot for ADCHWT OFDM and DFT OFDM.

Table 2. Processing simplicity.

\begin{tabular}{|c|c|c|c|}
\hline OFDM system & $\begin{array}{l}\text { Computational complexity in } \\
\text { terms of multiplications }\end{array}$ & Processing simplicity & $\begin{array}{l}\text { Execution } \\
\text { time (sec) }\end{array}$ \\
\hline $\begin{array}{l}\text { DWT /IDWT } \\
\text { with Haar WT }\end{array}$ & $\begin{array}{c}\mathrm{LN} \log _{2} \mathrm{~N} \\
(\mathrm{~L}=\text { length of filter })\end{array}$ & $\begin{array}{c}\text { Decimation, interpolation, } \\
\text { filtering }\end{array}$ & 0.181 \\
\hline $\begin{array}{l}\text { ADCHWT } \\
\text { uses DCT/IDCT }\end{array}$ & $\mathrm{N} \log _{2} \mathrm{~N}$ for each tree & No above processing steps & 0.140 \\
\hline
\end{tabular}

Though ADCHWT OFDM needs two real DCHWT trees for computation, complex processing steps employed by time domain WT OFDM are not required. Subband decomposition is achieved by grouping of scales and inverse of this group results in decimated signals. It requires mere concatenation of scales, in frequency domain for synthesis.

\section{Conclusion}

A new ADCHWT OFDM system is proposed for BPSK modulated signal and it shows remarkable improvement in performance in terms of BER compared to DFT OFDM and is comparable with Haar WT OFDM. PAPR performance shows $3 \mathrm{~dB}$ improvement than DFT OFDM and $0.3 \mathrm{~dB}$ than Haar WT OFDM. It could be noted that BER performance is preserved with an improvement in PAPR without any additional processing steps.

Improvement in performance is achieved due to advantage of operations in DCT domain, as there is no leakage effect; also, no smoothing window is required. Improved bandwidth utilization due to reduced overheads of $\mathrm{CP}$ and enhancement in BER, PAPR performance aided with shift invariance property of ADCHWT could position the proposed method as a preferred choice for transmission in fading channels. Suggested future work could be to explore shift invariance of ADCHWT in different fading channels. 


\section{References}

Lakshmanan M K and Nikookar H 2006 A Review of Wavelets for Digital Wireless Communications. Wireless Personal Communications, springer

Michael Bauer, René Anselment and Klaus Dostert 2009 Integrated Wavelet Packet Modulation and Signal Analysis Using analytic Wavelet Packets. 978-1-4244-3790-0/09, IEEE

Micheal C Jeruchim, Phillip Balaban and K Sam Shanmugan 2000 Simulation of communication systems Modelling, Methodology and techniques. Information Technology: Transmission, Processing and Storage, Kluwer Academic publisher, $2^{\text {nd }}$ Edition

Mirghani R and Ghavami M 2008 Comparison between Wavelet-based and Fourier-based Multicarrier UWB Systems. IET Communications 2(2): 353-358

Mohamed H M Nerma, Nidal S Kamel and Varun Jeoti 2009 OFDM based on complex wavelet transform. Signal Processing: An International Journal (SPIJ), Volume (3) Issue (2)

Narasimhan S V, Harish M, Haripriya A R and Nandini Basumallick 2008 Discrete cosine harmonic Wavelet transform and its application to signal compression and subband spectral estimation using modified group delay. Signal, Image and Video Processing 3(1): 85-99

Narasimhan S V, Nandini Basumallick and S Veena 2012 Introduction to wavelet transforms. A signal processing approach. Narosa publishing House

Ramjee Prasad 2004 OFDM for Wireless Communications Systems. Artech House, ISBN 1-58053-796-0

Selesnick I W, Baraniuk R G and Kinsbury N G 2005 The dual tree complex wavelet transform. IEEE Signal Processing Magazine, pp. 123-151

Shivamurti M and Narasimhan S V 2010 Analytic discrete cosine harmonic wavelettransform (ADCHWT) and its Applicationtosignal/image denoising. 978-1-4244-7138-6/10, (CIEEE

Suma M N, Narasimhan S V and Kanmani B 2012 The OFDM system based on Discrete Cosine Harmonic Wavelet Transform -National Communications Conference -NCC2012

Thomas Weickert, Claus Benjaminsen and UweKiencke 2009 Analytic Wavelet Packets-Combining the Dual-Tree Approach With Wavelet Packets for Signal Analysis and Filtering. IEEE Transactions on Signal Processing 57(2): 493-502 